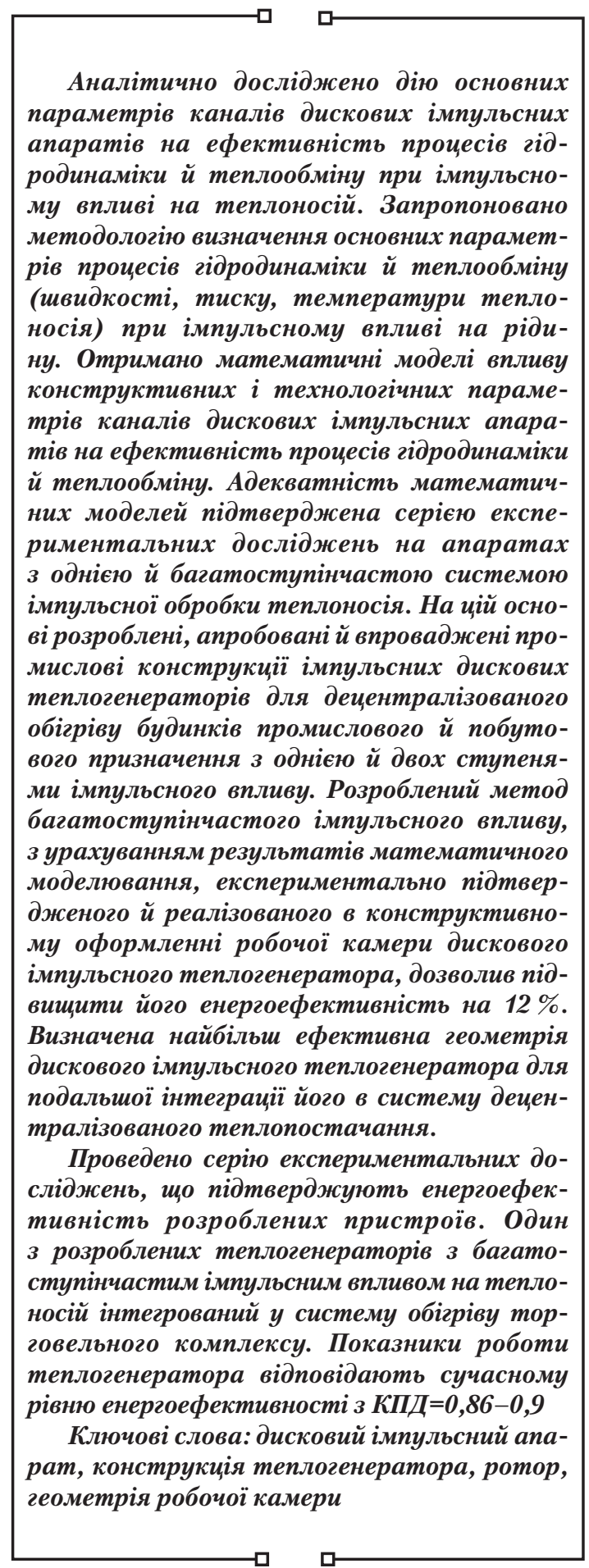

UDC 532.135

DOI: $10.15587 / 1729-4061.2019 .174629$

\section{ANALYTICAL AND EXPERIMENTAL STUDIES INTO THE PROCESSES OF HYDRODYNAMICS AND HEAT EXCHANGE IN THE CHANNELS OF DISK PULSE DEVICES}

V. Nikolsk y

Doctor of Technical Sciences, Professor Department of Energetic*

E-mail:vnikols1@gmail.com

I. Kuz y a ye v

Doctor of Technical Sciences, Professor

Department of Mechanical Engineering and Mechanical Engineering*

O. A lieks androv

$\mathrm{PhD}$, Senior Lecturer

Department of Applied Mechanics*

V. Ved

Senior Lecturer

Department of Equipment of Chemical Plants*

A. Pug a c h

Doctor of Science in Public Administration, PhD, Associate Professor

Department of Agricultural Machinery

Dnipro State Agrarian and Economic University Serhiya Efremova str., 25, Dnipro, Ukraine, 49600

V. Yaris

$\mathrm{PhD}$, Associate Professor, Director LLC Soyuztechnoprom Production Co. Kirova ave., 91, Dnipro, Ukraine, 49054

S. P t i t s y n

$\mathrm{PhD}$, Associate Professor, Technical Director

Ukrainian-Turkmen educational center «Erkin» D. Yavornytskoho ave., 94, Dnipro, Ukraine, 49038

V. Lopatin

Doctor of Technical Sciences, Senior Researcher Department of Mining Thermoaero Dynamics and Automated Systems Institute of Geotechnical Mechanics named by N. Poljakov

of National Academy of Sciences of Ukraine

Simferopolska str., 2-a, Dnipro, Ukraine, 49005

*Ukrainian State University of Chemical Technology

Gagarina ave., 8, Dnipro, Ukraine, 49005

\section{Introduction}

In current conditions, great attention is paid to finding new environmentally safe and effective ways to generate energy. Emphasis in this case is on the affordability and reproducibility of energy raw materials, environmental safety, economic efficiency, industrial feasibility, reliability, easy fabrication and operation of the equipment.
Pulse treatment of technological liquids is used mainly as a means of intensification of hydrodynamic and mass transfer processes. Currently, devices with the pulse effect on a liquid are employed as thermal power generators [1,2]. The working space of such types of assemblies represents a disk channel, inside which one disk is rotated while the second remains stationary. Such a configuration of the working space is typical for a significant number of equipment, for example: 
centrifugal pumps, circular extruders, rotary cavitation units, and other devices.

The flow of a classic viscous fluid in the region between rotating coaxial disks has been studied by many authors motivated by the fact that these tasks are not only of theoretical but also of great practical interest.

Intensification of the hydrodynamical and heat transfer processes in the disk pulse devices, as a consequence of the optimization of their workspace, is essential to improve existing, as well as construct new, energy-efficient thermal machines with high energy efficiency [3-5].

A growing body of research related to improving energy efficiency of disk devices based on the pulse treatment of a heat-carrier using renewable environmentally-friendly sources of energy is one of the most important fields in the development of energy complex.

\section{Literature review and problem statement}

Disk pulse devices are interesting to researchers due to the possibility of their wide application for the pulse treatment of a heat-carrier in heating systems [6]. Current research in the field of improvement of pulse devices is aimed at the optimization of technological parameters by changing the geometric dimensions of the working chamber. Of special interest to researchers is to study the hydrodynamics and heat exchange in the pulsed device under different modes of operation.

Paper [7] reports an analytic description of hydrodynamic processes in the working chambers of pulse devices of various designs, based on the equation of a fluid motion along a channel in the vector form. In [8], authors give a continuity equation for compressible media. The solutions obtained represent particular cases, adopted with many assumptions that do not apply to all the designs of pulsed devices.

In [9], authors have attempted to describe the hydrodynamic processes under non-isothermal conditions in a cylindrical coordinate system. They derived continuity equations for an incompressible fluid and a heat balance equation of energy in the chamber using a function of dissipation $\left(F_{d i s}\right)$ :

$$
\begin{aligned}
& \frac{1}{r} \cdot \frac{\partial}{\partial r}\left(r \cdot V_{r}\right)+\frac{1}{r} \cdot \frac{\partial V_{\varphi}}{\partial \varphi}+\frac{\partial V_{z}}{\partial z}=0, \\
& \rho \cdot C_{p} \cdot\left(\frac{\partial T}{\partial t}+V_{r} \cdot \frac{\partial T}{\partial r}+\frac{V_{\varphi}}{r} \cdot \frac{\partial T}{\partial \varphi}+V_{z} \cdot \frac{\partial T}{\partial z}\right)= \\
& =-\left(\frac{\partial q_{r}}{\partial r}+\frac{1}{r} \cdot \frac{\partial q_{\varphi}}{\partial \varphi}+\frac{\partial q_{z}}{\partial z}+\frac{q_{r}}{r}\right)+F_{d i s} .
\end{aligned}
$$

A detailed analysis of the resulting form of the equation reveals that the expression represents a Navier-Stokes equation [10], solved in the cylindrical coordinate system, which was obtained by Karmann for a Newtonian fluid and a rotating disk [11, 12].

Applying the results from work [13], authors of [14] made the problem more difficult and derived a solution to the equation of fluid motion between two disks. When two disks rotate under condition of constancy for angular velocity and contrary directions, the mass of the fluid, which is outside the boundary layers of the disks would not spin [15].

Papers [16-18] investigated different models of the multi-sectional movement of a fluid, which are characterized by dependence on the values for a Reynolds number. In this case, the equations of motion were consistent with the form given in works [12-14]. The proof of existence of multiple solutions is a breakthrough in the research into the hydrodynamics of a fluid in a disk space.

Study [19] reports a motion model of the Maxwell's fluid in a disk chamber taking into consideration the properties of a viscous elasticity. The authors showed the transition of the equation obtained into the equation for Newtonian fluids under a stationary current mode.

A detailed analysis of solutions to the problems on the movement of a fluid taking into consideration the assigned boundary conditions for disk chambers, given in papers [12-19] indicates that the results obtained do not qualify for universal use when designing disk chambers for pulse devices [20].

We did not find any motion models of a liquid or solutions to the equation of motion for the case when the process is close to adiabatic, which is characterized by the accumulation of heat in the working chamber of the device inherent in actual conditions. This makes it a priority task to construct mathematical models for defining the main parameters for the processes of fluid dynamics and heat exchange under the pulse impact on a liquid when designing modern energy-efficient pulsed devices.

\section{The aim and objectives of the study}

The aim of this study is to study analytically the processes of hydrodynamics and heat exchange between contact phases in the system liquid-solid body using the example of disk pulse devices with external sources of energy.

To accomplish the aim, the following tasks have been set:

- to perform analytical study into the influence of basic parameters of channels in disk pulse devices on the effectiveness of processes of hydrodynamics and heat exchange under the pulse impact on a heat-carrier;

- to define optimal geometrical characteristics for a disk pulse heat generator in terms of its energy efficiency;

- to design a structure of the experimental disk pulse device with an energy-efficient working chamber.

\section{Analytical study into the processes of hydrodynamics and heat exchange in a working channel of the disk pulse device and their computer implementation}

In order to carry out an analytical study into the influence of basic geometric parameters of the disk pulse device's channel on the processes of hydrodynamics and heat exchange, to build relevant mathematical models, we have designed a structure for the disk pulse device's working chamber and, based on it, an estimation scheme (Fig. 1). In this case, we accepted the following assumptions:

- the process in a disk pulse device is axisymmetric; we disregard changes in the physical quantities along the angular coordinate $(\partial / \partial \varphi=0)$;

- the process is stationary (changes in the physical quantities over time are not significant $\partial / \partial t=0)$;

- mass forces are neglected $\left(\rho \cdot g_{i}=0\right)$;

- the component of speed $V_{z}$ is neglected since $h \ll R_{n}$;

- we do not consider the temperature gradient due to heat conductivity along the $r$ axis and the convective heat transfer along the $z$ axis. 


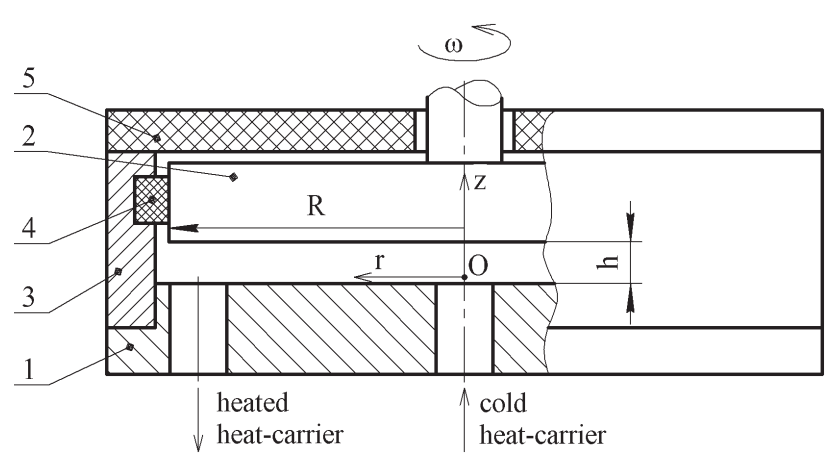

Fig. 1. Estimation scheme for building mathematical models: 1 - fixed disk; 2 - rotating disk; 3 - body; 4 - sealing ring; 5 - heat insulating element

Considering the first three assumptions, the motion equations of a liquid along a disk channel in the cylindrical coordinate system, taking into consideration the deformation velocity tensor component [21], will take the form, for a Newtonian fluid:

$$
\begin{aligned}
& \rho \cdot\left(V_{r} \cdot \frac{\partial V_{r}}{\partial r}-\frac{V_{\varphi}^{2}}{r}\right)= \\
& =-\frac{\partial P}{\partial r}+\eta \cdot\left[2 \cdot \frac{\partial^{2} V_{r}}{\partial r^{2}}+\frac{\partial^{2} V_{r}}{\partial z^{2}}+\frac{2}{r} \cdot\left(\frac{\partial V_{r}}{\partial r}-\frac{V_{r}}{r}\right)\right] ; \\
& \rho \cdot\left(V_{r} \cdot \frac{\partial V_{\varphi}}{\partial r}+\frac{V_{r} \cdot V_{\varphi}}{r}\right)=\eta \cdot\left[\frac{\partial^{2} V_{\varphi}}{\partial r^{2}}+\frac{1}{r} \cdot \frac{\partial V_{\varphi}}{\partial r}-\frac{V_{\varphi}}{r^{2}}+\frac{\partial^{2} V_{\varphi}}{\partial z^{2}}\right] ; \\
& 0=-\frac{\partial P}{\partial z}+\eta \cdot\left(\frac{\partial^{2} V_{r}}{\partial z \partial r}+\frac{1}{r} \cdot \frac{\partial V_{r}}{\partial z}\right) .
\end{aligned}
$$

Continuity equation in the cylindrical system (1) is:

$$
\frac{\partial V_{r}}{\partial r}+\frac{V_{r}}{r}=0 .
$$

The latter expression produces the following ratio:

$$
V_{r}=\frac{f(z)}{r} .
$$

Assuming the angular component of velocity is proportional to the radius:

$$
V_{\phi}=\varsigma(z) \cdot r .
$$

In expressions (7), (8), $f(z)$ and $\varsigma(z)$ are the functions that depend on coordinate $z$.

Substituting (7), (8) in expressions (3), (4) leads to the system:

$$
\begin{aligned}
& \rho \cdot\left(-\frac{f(z)^{2}}{r^{3}}-\varsigma(z)^{2} \cdot r\right)=-\frac{\partial P}{\partial r}+\eta \cdot \frac{f^{\prime \prime}(z)}{r} ; \\
& \rho \cdot \frac{2 \cdot f(z)}{r} \cdot \varsigma(z)=\eta \cdot \varsigma^{\prime \prime}(z) \cdot r \\
& 0=-\frac{\partial P}{\partial z} .
\end{aligned}
$$

We neglect a change in pressure along the radial axis at head-free flow, when a pressure gradient along the $z$ axis is zero. Finally, the system of equations for functions $f(z)$ and $\varsigma(z)$ will be given in the form:

$$
\begin{aligned}
& \eta \cdot \frac{f^{\prime \prime}(z)}{r}+\rho \cdot\left(\frac{f(z)^{2}}{r^{3}}+\varsigma(z)^{2} \cdot r\right)=0 ; \\
& \eta \cdot \varsigma^{\prime \prime}(z) \cdot r-\rho \cdot \frac{2 \cdot f(z)}{r} \cdot \varsigma(z)=0 .
\end{aligned}
$$

The mathematical models were implemented using the mathematical software Mathcad 11, applying the computational unit Given-Odesolve. The accepted boundary conditions were the following parameters: viscosity coefficient of a fluid $\eta=5.471 \cdot 10^{-4} \mathrm{~Pa} \cdot \mathrm{s}$; the angular velocity of disk rotation $\omega=6.0 \mathrm{~s}^{-1}$; a liquid's density $\rho=988.1 \mathrm{~kg} / \mathrm{m}^{3}$; the height of a disk channel $h=(5 \div 12) \cdot 10^{-3} \mathrm{~m}$; the radius of a disk $r=0.11 \div 0.17 \mathrm{~m}$.

Fig. 2, 3 show the estimation dependences from the constructed mathematical models.
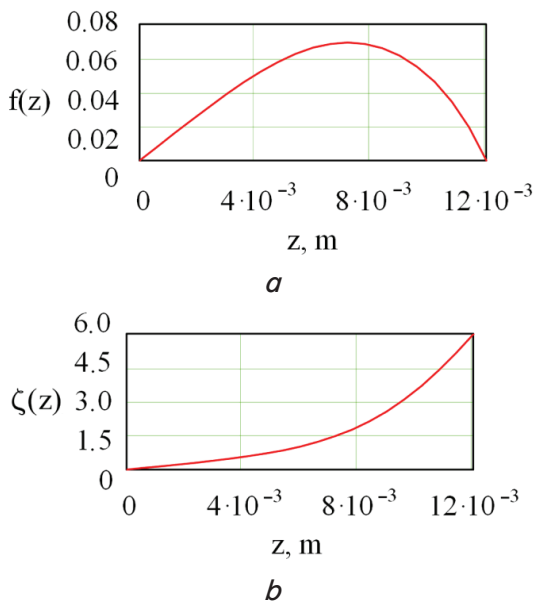

Fig. 2. Estimation dependences from the constructed mathematical models: $a$ - function $f(z) ; b-$ function $\varsigma(z)$
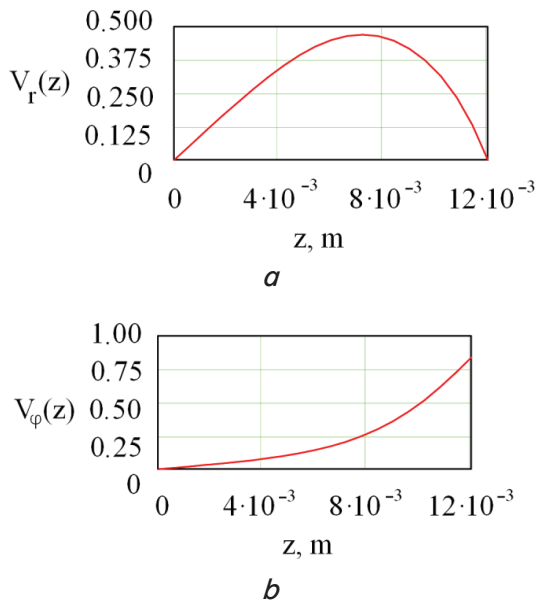

Fig. 3. Estimation dependences for velocity components: $a-V_{r}(z) ; b-V_{\varphi}(z)$

Fig. 4 shows the velocity components dependences at different values of radii. 


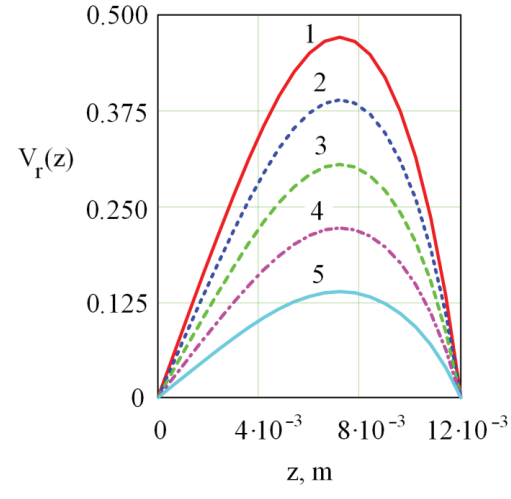

$a$

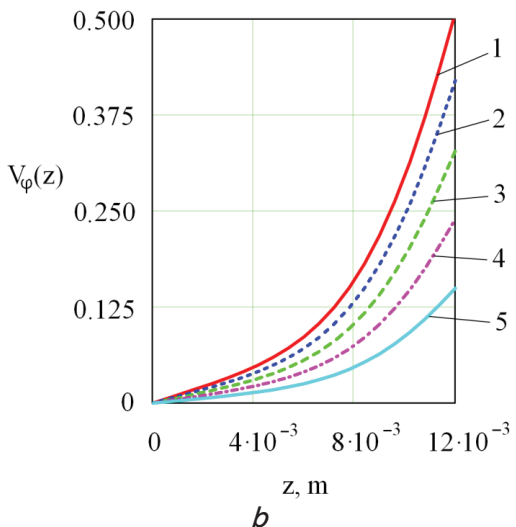

b

Fig. 4. Estimated values for velocity components at different radii: $1-r=0.17 \mathrm{~m} ; 2-r=0.14 \mathrm{~m} ; 3-r=0.11 \mathrm{~m} ; 4-r=0.08 \mathrm{~m} ; 5-r=0.05 \mathrm{~m}$ :

$a$-value for component $V_{r}(z) ; b-$ value for component $V_{\varphi}(z)$

In further calculations, in order to apply the results obtained, we shall approximate velocity components $V_{r}(z)$ and $V_{\varphi}(z)$. The approximation is based on the generalized regression function linfit from the software package Mathcad.

Fig. 5, 6 show the results of approximating the radial $V_{r}$ and tangential $V_{\varphi}$ velocity components.

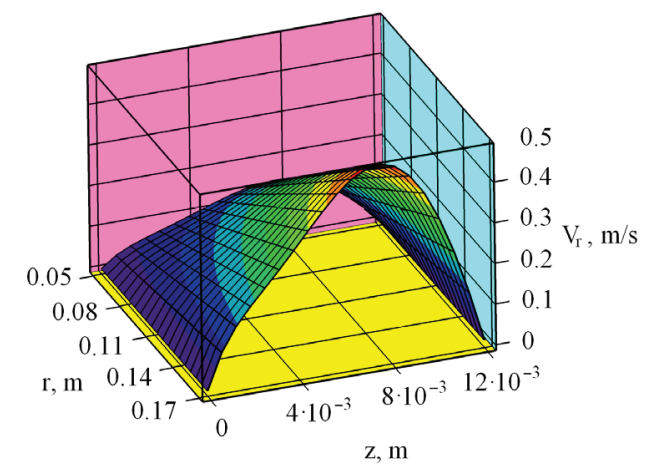

Fig. 5. Approximating 3D diagram for the velocity radial

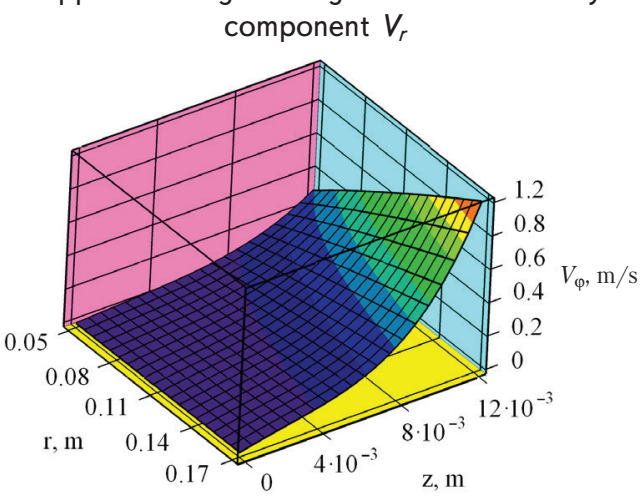

Fig. 6. Approximating 3D diagram for the velocity tangential component $V_{\varphi}$

Approximating expressions for velocity components are recorded in the analytical dependences:

- for the radial component:

$V_{r}(r, z)=k 1 \cdot r \cdot z \cdot \exp (c \cdot z)+k 2 \cdot r \cdot z \cdot \sin (\pi \cdot z / h) ;$

- for the tangential component:

$$
\begin{aligned}
& V_{\varphi}(r, z)=\left(k 1_{0} \cdot r^{b 1}+k 1_{1} \cdot r^{d 1}\right) \cdot z \cdot \exp (c 1 \cdot z)+ \\
& +\left(k 2_{0} \cdot r^{b 1}+k 2_{1} \cdot r^{d 1}\right) \cdot z^{a 1} .
\end{aligned}
$$

The coefficients included in the last expressions accept the following values: $k 1=$ $=1.038 \cdot 10^{4}, k 2=3.824 \cdot 10^{3}, c=-5,000, k 1_{0}=$ $=-1.284 \cdot 10^{3}, k 1_{1}=2.598 \cdot 10^{4}, k 2_{0}=-1.794 \cdot 10^{12}$, $k 2_{1}=3.628 \cdot 10^{12}, c 1=-585.4, a 1=4$.

To derive the mathematical models describing a temperature field in the disk channel, we use expression (2) by applying the dissipation function $F_{d i s}$ in the form:

$$
\begin{aligned}
& F_{d i s}=\sigma_{r r} \cdot \frac{\partial V_{r}}{\partial r}+\frac{\sigma_{\varphi \varphi}}{r} \cdot\left(\frac{\partial V_{\varphi}}{\partial \varphi}+V_{r}\right)+ \\
& +\sigma_{z z} \cdot \frac{\partial V_{z}}{\partial z}+\sigma_{r \varphi} \cdot\left(\frac{\partial V_{\varphi}}{\partial r}+\frac{1}{r} \cdot \frac{\partial V_{r}}{\partial \varphi}-\frac{V_{\varphi}}{r}\right)+ \\
& +\sigma_{\varphi z} \cdot\left(\frac{1}{r} \cdot \frac{\partial V_{z}}{\partial \varphi}+\frac{\partial V_{\varphi}}{\partial z}\right)+ \\
& +\sigma_{z r} \cdot\left(\frac{\partial V_{r}}{\partial z}+\frac{\partial V_{z}}{\partial r}\right) .
\end{aligned}
$$

These equations, taking into consideration the assumptions introduced, are recorded, accordingly:

$$
\begin{aligned}
& \rho \cdot C_{p} \cdot V_{r} \cdot \frac{\partial T}{\partial r}=-\frac{\partial q_{z}}{\partial z}+F_{d i s}, \\
& F_{d i s}=\sigma_{r r} \cdot \frac{\partial V_{r}}{\partial r}+\sigma_{\varphi \varphi} \cdot \frac{V_{r}}{r}+ \\
& +\sigma_{r \varphi} \cdot\left(\frac{\partial V_{\varphi}}{\partial r}-\frac{V_{\varphi}}{r}\right)+\sigma_{\varphi z} \cdot \frac{\partial V_{\varphi}}{\partial z}+\sigma_{z r} \cdot \frac{\partial V_{r}}{\partial z} .
\end{aligned}
$$

Equation (18), in addition to velocity components $V_{r}$ and $V_{\varphi}$, includes their deformations $-\partial V_{r} / \partial r$ and $\partial V_{r} / \partial z$, $\partial V_{\varphi} / \partial r$ and $\partial V_{\varphi} / \partial z$. By differentiating them and introducing a simplifying notation, we obtain the following values of deformations:

$$
\begin{aligned}
& \frac{\partial V_{r}}{\partial r}=d V_{r r}(r, z)=z \cdot\left[k 1 \cdot \exp (c \cdot z)+k 2 \cdot \sin \left(\frac{\pi \cdot z}{h}\right)\right] \\
& \frac{\partial V_{r}}{\partial z}=d V_{r z}(r, z)=k 1 \cdot r \cdot \exp (c \cdot z) \cdot(1+c \cdot z)+ \\
& +k 2 \cdot r \cdot\left[\sin \left(\frac{\pi \cdot z}{h}\right)+\frac{\pi \cdot z}{h} \cdot \cos \left(\frac{\pi \cdot z}{h}\right)\right] \\
& \frac{\partial V_{\varphi}}{\partial r}=d V_{\varphi r}(r, z)= \\
& =\left(k 1_{0} \cdot b 1 \cdot r^{b 1-1}+k 1_{1} \cdot d 1 \cdot r^{d 1-1}\right) \cdot z \cdot \exp (c 1 \cdot z)+ \\
& +\left(k 2_{0} \cdot b 1 \cdot r^{b 1-1}+k 2_{1} \cdot d 1 \cdot r^{d 1-1}\right) \cdot z^{a 1} \\
& \frac{\partial V_{\varphi}}{\partial z}=d V_{\varphi z}(r, z)= \\
& =\left(k 1_{0} \cdot r^{b 1}+k 1_{1} \cdot r^{d 1}\right) \cdot \exp (c 1 \cdot z) \cdot(1+z \cdot c 1)+ \\
& +\left(k 2_{0} \cdot r^{b 1}+k 2_{1} \cdot r^{d 1}\right) \cdot z^{a 1-1} \cdot a 1 .
\end{aligned}
$$

Taking into consideration the rheological equation of state for a Newtonian fluid, we shall rewrite equation (18) as follows: 


$$
F_{\text {dis }}(r, z)=\eta \cdot\left[\begin{array}{l}
f 1(r, z)+f 2(r, z)+ \\
+f 3(r, z)+f 4(r, z)+f 5(r, z)
\end{array}\right] ;
$$

where

$$
\begin{aligned}
& f 1(r, z)=2 \cdot\left(\frac{\partial V_{r}}{\partial r}\right)^{2} ; \\
& f 2(r, z)=\left(\frac{V_{r}}{r}\right)^{2} ; \\
& f 3(r, z)=2 \cdot\left(\frac{\partial V_{\varphi}}{\partial r}-\frac{V_{\varphi}}{r}\right)^{2} ; \\
& f 4(r, z)=2 \cdot\left(\frac{\partial V_{\varphi}}{\partial z}\right)^{2} ; \\
& f 5(r, z)=2 \cdot\left(\frac{\partial V_{r}}{\partial z}\right)^{2} .
\end{aligned}
$$

A 3D diagram for the dissipation function is shown in Fig. 7.

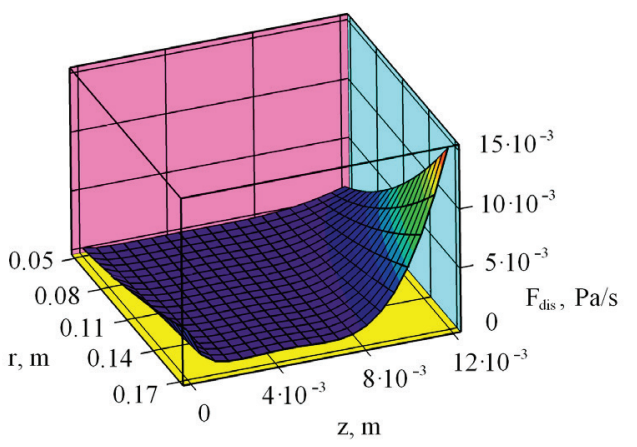

Fig. 7. 3D diagram for the dissipation function $F_{\text {dis }}$

Dissipation function can be used to determine the power that is consumed in the working area of any geometric configuration, including a disk channel. To this end, it is necessary to integrate the dissipation function for the volume of a working zone. If a working area is of a regular shape (for the case of a disk channel), the volumetric channel can be represented in the form of a triple integral, and the triple integral - as a double one, provided the symmetry relative to the angular coordinate. Then, to determine the power in a disk channel, the following expression holds:

$$
W_{d k}=2 \cdot \pi \cdot \int_{0}^{h} \int_{R_{1}}^{R_{2}} F_{d i s}(r, z) \cdot r \mathrm{~d} r \mathrm{~d} z
$$

where $R_{1}, R_{2}$ are, respectively, the initial and final radii of a disk.

Dissipation function (23), considering dependences (18) to (22), is cumbersome in terms of the ratios it includes. To simplify the application of this expression when determining a temperature field from equation (17), we shall approximate it (similar to the approximation of velocity components). Then the approximating expression for the dissipation function takes the form:

$$
F_{\text {disA }}=r^{b 2} \cdot k 1 \cdot \exp (a 2 \cdot z) .
$$

For the examined case, the coefficients appearing in the last expression accept the following values: $a 2=2, b 2=3.3$, $c 2=4,000, d 2=100$.
We shall rewrite the thermal energy balance equations (17) considering a Fourier law of heat flow $q_{z}=-\lambda \cdot \partial T / \partial z$, where $\lambda$ is a thermal conductivity coefficient:

$$
\rho \cdot C_{p} \cdot V_{r} \cdot \frac{\partial T}{\partial r}=\lambda \cdot \frac{\partial^{2} T}{\partial z^{2}}+F_{d i s A} .
$$

The analytical solution to equation (26) is derived by applying a method of the integral Laplace transform [21, 22].

In this case, one must have two boundary conditions along the $z$ coordinate and one initial condition along the radial coordinate $r$.

With respect to the initial condition along the $r$ coordinate we write:

$$
T=T_{n} \text { at } r=R_{1},
$$

where $T_{n}$ is the initial temperature at the inlet to a working channel.

Along the $z$ coordinate, in a general case, there apply the boundary conditions of orders I, II, and III:

- boundary condition of order I; when one assigns temperature $T_{\varkappa}$ :

$$
T=T_{w} \text { at } x_{i}=W,
$$

where $x_{i}$ is the generalized coordinate; $W$ is the characteristic dimension;

- boundary condition of order II; when one assigns the heat flow $q_{w}$ :

$$
\pm \lambda \cdot \frac{\partial T}{\partial x_{i}}=q_{w} \text { at } x_{i}=W
$$

- boundary condition of order III; when one assigns, along the surface of the channel, convection heat exchange with the environment:

$$
\pm \lambda \cdot \frac{\partial T}{\partial x_{i}}=\alpha_{T} \cdot\left(T_{c}-T\right) \text { at } x_{i}=W
$$

where $T_{c}$ is the ambient temperature; $\alpha_{T}$ is the heat transfer coefficient.

The plus and minus signs correspond to the direction of the coordinate axis and a heat flux vector, when directions match - plus, otherwise - minus.

For boundary conditions of order II with respect to ratio (29), for the case of a thermally insulated surface, one can write:

$$
\frac{\partial T}{\partial x_{i}}=0 \text { at } x_{i}=W
$$

For a fixed disk, given a possibility of structural execution of a thermal stabilization system, one can apply the first-kind boundary condition, and for a rotating disk, subject to its thermal insulation, one should use ratio (31). Then the boundary conditions along the $z$ coordinate are recorded in the form:

$$
\left.\begin{array}{l}
T=T_{k} \text { at } z=0 \\
\frac{\partial T}{\partial z}=0 \text { at } z=h
\end{array}\right\}
$$

where $T_{k}$ is the body's temperature from the side of a liquid. 
In addition to the temperature, used for the Laplace transform, there are two functions that depend on coordinate $r$, namely the velocity component $V_{r}$ and the dissipation function $F_{\text {disA }}$.

When deriving the analytical solution, we used the procedure proposed in $[23,24]$. Fixed values for the velocity component $V_{r}$ and $F_{d i s}$ along the $r$ coordinate, corresponding to their averaged values along the $z$ coordinate, shall be denoted by $V_{r c}$ and $F_{d c}$. The latter assumption holds if calculation is performed in steps along the radial coordinate. In this case, at each step, the initial value for temperature is adjusted. Thus, the resulting value for temperature at the preceding step becomes the initial value at the subsequent step.

Then equation (26) can be written as:

$$
\rho \cdot C_{p} \cdot V_{r c} \cdot \frac{\partial T}{\partial r}=\lambda \cdot \frac{\partial^{2} T}{\partial z^{2}}+F_{d c} \cdot
$$

Upon the integral Laplace transform along the $r$ coordinate, equation (33) takes the form:

$$
\rho \cdot C_{p} \cdot V_{r c} \cdot[s \cdot \Phi-T(0)]=\lambda \cdot \frac{d^{2} \Phi}{d z^{2}}+\frac{F_{d c}}{s},
$$

where $\vartheta$ is the temperature image $T$; s is the variable of a Laplace transform; $T(0)$ is the initial temperature value (considering notation in (27), we obtain $\left.T(0)=T_{n}\right) ; \Gamma(b 2+1)$ is the gamma function.

We rewrite expression (34) as follows:

$$
\frac{d^{2} \Phi}{d z^{2}}-\frac{V_{r c}}{a} \cdot s \cdot \Phi=-\frac{V_{r c}}{a} \cdot T_{n}-\frac{F_{d c}}{s}
$$

where $a$ is the coefficient of thermal diffusivity $\left(a=\lambda /\left(\rho \cdot C_{p}\right)\right)$.

Expression (35) is a differential equation in ordinary derivatives, its solution is recorded by analogy [34].

$$
\Phi=\varphi_{2} \cdot \int \frac{\varphi_{1} \cdot h}{W} \mathrm{~d} z+\varphi_{1} \cdot \int \frac{\varphi_{2} \cdot h}{W} \mathrm{~d} z+C_{1} \cdot \varphi_{1}+C_{2} \cdot \varphi_{2},
$$

where

$$
\begin{aligned}
& \varphi_{1}=\exp \left(\frac{\lambda_{k}}{2} \cdot z\right) ; \quad \varphi_{2}=\exp \left(-\frac{\lambda_{k}}{2} \cdot z\right) ; \\
& W=\varphi_{1} \cdot \frac{d \varphi_{2}}{d z}-\varphi_{2} \cdot \frac{d \varphi_{1}}{d z}, \quad \lambda_{k}= \pm 2 \cdot \sqrt{V_{r c} / a} \cdot \sqrt{s} .
\end{aligned}
$$

Upon completion of the required transformations, equation (36) is reduced to the form:

$$
\Phi=\frac{T_{n}}{s}+\frac{F_{d c} \cdot a}{s^{2} \cdot V_{r c}}+\left(T_{k}-T_{n}\right) C H(s, z)-\frac{F_{d c} \cdot a}{s \cdot V_{r c}} \cdot C H(s, z),
$$

where

$$
C H(s, z)=\frac{\cosh \left[\sqrt{\frac{V_{r c}}{a}} \cdot \sqrt{s} \cdot(h-z)\right]}{s \cdot \cosh \left[\sqrt{\frac{V_{r c}}{a}} \cdot \sqrt{s} \cdot h\right]} .
$$

The integration constants $C_{1}$ and $C_{2}$ were determined considering the boundary conditions (32):

$$
\left.\begin{array}{l}
\Phi=\frac{T_{k}}{s} \text { at } z=0 \\
\frac{\partial \Phi}{\partial z}=0 \text { at } z=h .
\end{array}\right\}
$$

To derive expressions for the temperature field distribution in the original, one must find the originals of each of the complexes in the right-hand side of equation (37).

Detailed transition from the image to the original for equations in the form (37) is given in paper [11].

Finally, in the original, the distribution of a temperature field in the disk channel can be represented as follows:

$$
T(r, z)=T_{k}+\frac{2 \cdot\left(T_{k}-T_{n}\right)}{\pi} \cdot F 1(r, z)-\frac{2 \cdot F_{d c} \cdot h^{2}}{\pi^{3}} \cdot F 2(r, z),
$$

where

$$
\begin{aligned}
& F 1(r, z)=\sum_{k} \frac{C S(k, z)}{k_{2} \cdot(-1)^{k}} \cdot E X(k, r) ; \\
& F 2(r, z)=\sum_{k} \frac{C S(k, z)}{k_{2}{ }^{3} \cdot(-1)^{k}} \cdot[1-E X(k, r)] ; \\
& C S(k, z)=\cos \left[\pi \cdot k_{2} \cdot\left(1-\frac{z}{h}\right)\right] ; \\
& E X(k, r)=\exp \left[-\frac{\pi^{2} \cdot a}{h^{2} \cdot V_{r c}} \cdot k_{2}{ }^{2} \cdot r\right] ; \\
& k_{2}=k-1 / 2 .
\end{aligned}
$$

We calculated a temperature field in line with the mathematical model (39) using the mathematical software Mathcad. The following conditions were adopted: $\rho=988 \mathrm{~kg} / \mathrm{m}^{3}$; $h=(5 \div 12) \cdot 10^{-3} \mathrm{~m} ; C_{p}=4,181 \mathrm{~J} /(\mathrm{kg} \cdot \mathrm{K}) ; \lambda=0.648 \mathrm{~W} /(\mathrm{m} \cdot \mathrm{K})$; $T_{n}=293 \mathrm{~K} ; T_{k}=333 \mathrm{~K}$.

The averaged values for $V_{r c}$ and $F_{d c}$ match expressions (14) and (25).

Fig. 8 shows a three-dimensional diagram of the temperature field distribution in a disk gap.

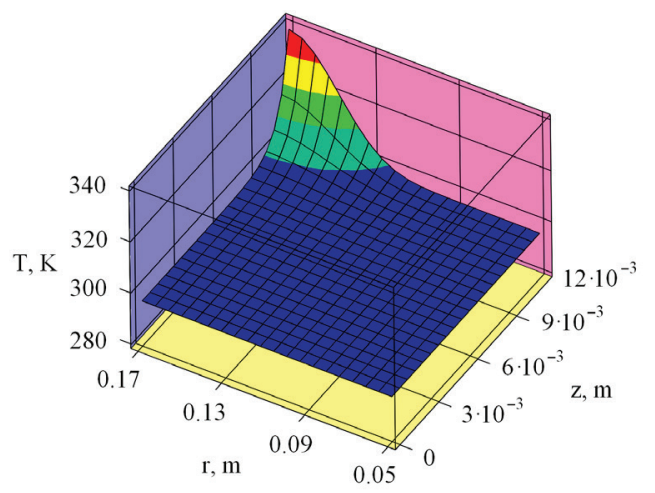

Fig. 8. 3D diagram of the temperature field distribution in a disk gap

Analysis of the temperature field distribution in a disk gap indicates that the maximum temperature of $330 \mathrm{~K}$ corresponds to the impeller's radius $r=0.16-0.17 \mathrm{~m}$ and the height of a disk channel (along the $z$ axis) $h=0.012 \mathrm{~m}$. 


\section{Description of the design features of the experimental disk pulse generator}

To verify adequacy of the constructed mathematical models, which define the influence of geometric parameters for the disk pulse devices' channels on efficiency of the processes of heat exchange and fluid dynamics, we have performed an experimental study into the flow of an incompressible fluid in the disk pulse device. To this end, we designed and manufactured a disk pulse heat generator (Fig. 9). The heat generator represents a working chamber with the varying geometry of disk channels and the working disk's diameter (a rotor's step). Based on it, we assembled an experimental bench equipped with a measurement system for testing operations (Fig. 10).

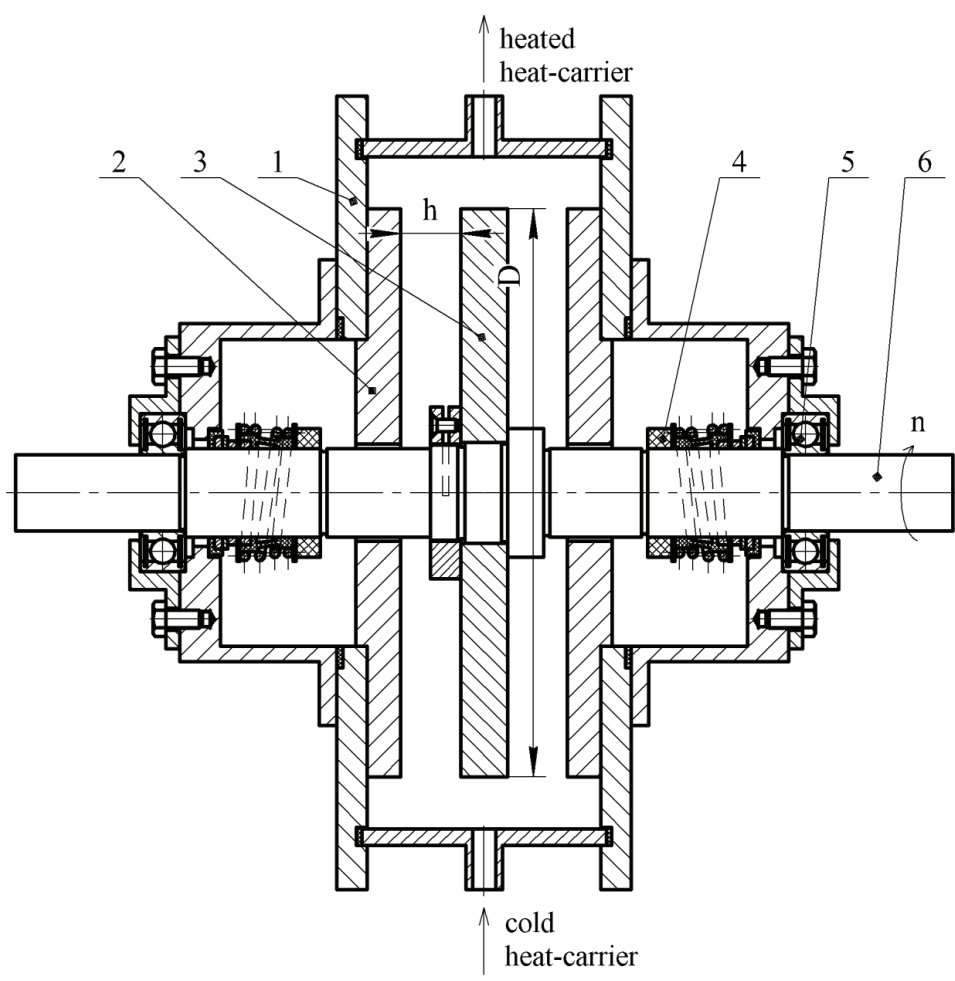

Fig. 9. Principal diagram of the designed disk pulse heat generator: 1 - stator's body; 2 - stator disk; 3 - working disk (stator's step); 4 - rotor seal; 5 - bearing; 6 - rotor shaft

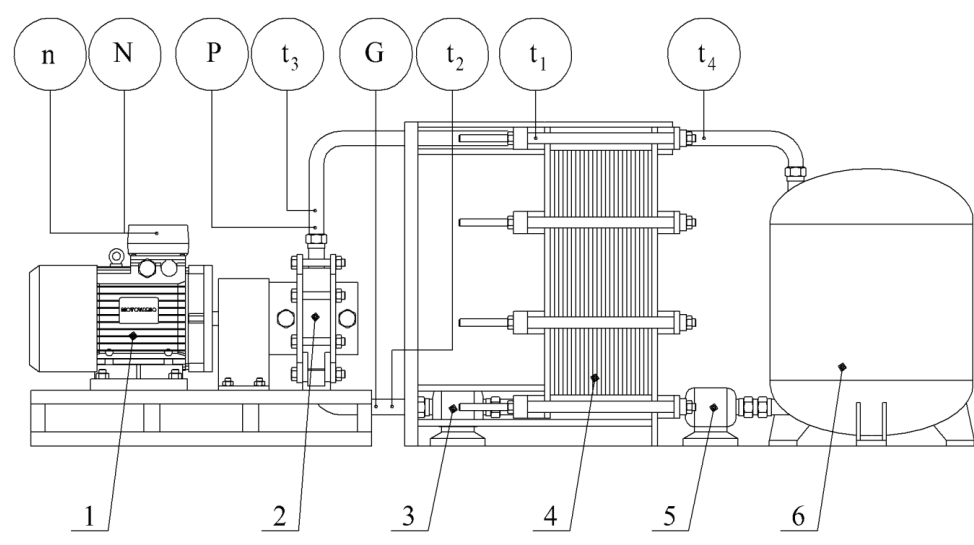

Fig. 10. Experimental bench for testing effectiveness of the disk pulse heat generator operation: 1 - electric motor; 2 - chamber of disk pulse heat generator; 3,5 - pump; 4 - plate heat exchanger; $6-$ accumulating capacity
A change in the number of disks mounted onto the rotor shaft (impellers) enabled the process of single-step and multi-step cavitation. Based on the systematic analysis of research results, we determined the most efficient geometry of a disk pulse heat generator in order to subsequently integrate it into the system of decentralized heating.

\section{Testing the disk pulse heat generator}

A procedure for testing the disk pulse heat generator for energy efficiency was consistent with the procedure described in [2].

In this case, we monitored the temperature, flow rate, pressure at the inlet and outlet of the heat generator, the power used, rotation number, consumption of the heated heat-carrier $G=0.27 \div 1.1 \mathrm{~kg} / \mathrm{s}$, installation power $N=7.5 \div 15 \mathrm{~kW} \cdot \mathrm{h}$; the number of revolutions of the rotor's rotation $n=2,500 \div 3,000 \mathrm{rpm}$ in accordance with the diagram in Fig. 10. Dimensions of the working chamber, the impeller's radius $r=0.17 \mathrm{~m}$ and the height of a disk channel $h=12 \cdot 10^{-3} \mathrm{~m}$, matched the maximum values for efficiency of the processes of hydrodynamics and heat exchange, derived on the basis of mathematical models (Fig. 5-8).

We treated the experimental data from 25 experiments (5-time experimental heating at each representative point under the selected boundary conditions) in line with the procedure from [25].

Fig. 11 shows the thermograms of heating energy efficient structures of the single-step and two-step disk pulse heat generators. Each point at a thermogram corresponds to the average indicators for five-time experimental heating at representative points. Approximation of the experimental points indicates a linear dependence of temperature on the heating time.

Based on the processed research results, efficiency (E) of a single-step disk heat generator (dependence 1) was $\eta=0.76$.

In order to enhance efficiency of the heat generator, we used a multi-stage pulse influence on a heat-carrier by mounting the second disk onto the rotor. A gap between the disks of $c=12 \cdot 10^{-3} \mathrm{~m}$ was established to match the highest indicators for efficiency of the processes of heat exchange and hydrodynamics, derived on the basis of mathematical models (Fig. 5-8).

Efficiency coefficient based on the results from processing the data from the study (dependence 2) amounted to $\eta=0.84$.

Thus, the method of a multi-stage pulse influence, taking into consideration the results of mathematical modeling, experimentally confirmed and implemented in the structural execution of the disk pulse heat generator's working chamber, has made it possible to improve its energy efficiency by $12 \%$.

A general view of the heat-generating installation with the designed heat generator with a two-stage pulse influence on a heat-carrier is shown in Fig. 12 


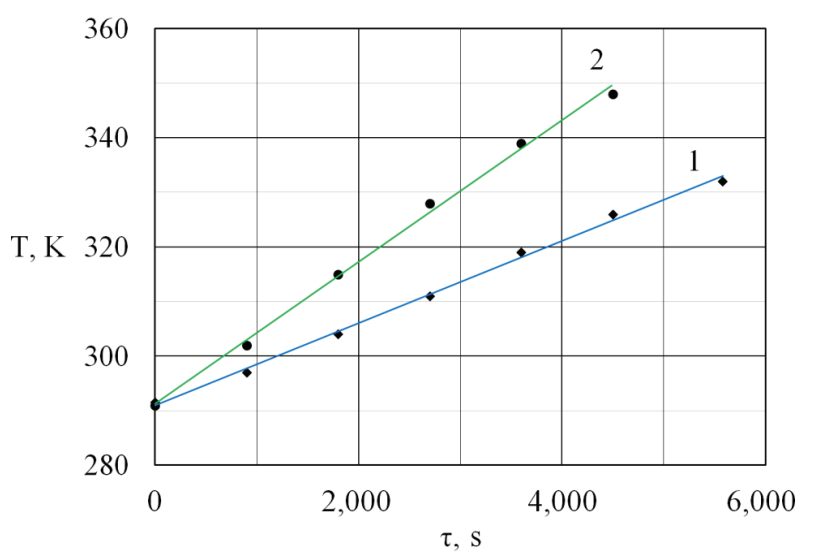

Fig. 11. Thermogram of heating a heat-carrier at a single-step -1 and a two-step -2 disk pulse heat generators

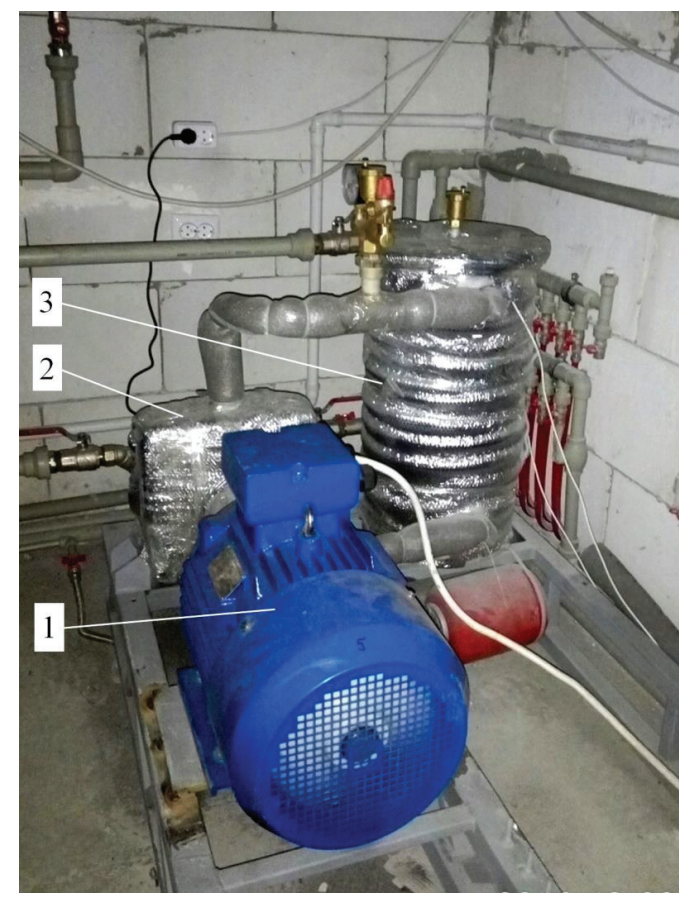

Fig. 12. General view of the heating system for a shopping mall with the integrated disk pulse heat generator: 1 - electric motor; 2 - disk pulse heat generator; 3 - heat exchanger

The designed heat generator was integrated into a heat supply system for a shopping mall with the total area of $2,700 \mathrm{~m}^{2}$

\section{Discussion of results of studying the designed disk pulse heat generator}

High energy efficiency of the designed heat-generating unit is due to the following features of its structure:

1. The optimization of geometrical parameters of its working chamber based on the results from mathematical modeling.

As can be seen from a diagram of the distribution of a temperature field in a disk gap (Fig. 8), the maximum temperature of $330 \mathrm{~K}$ is achieved when the impeller's radius is $r=0.17 \mathrm{~m}$ and the height of a disk channel is $h=12 \cdot 10^{-3} \mathrm{~m}$. At the same design parameters, we achieved the maximum value for the dissipation function $F_{d i s}=12 \cdot 10^{-3} \mathrm{~Pa} / \mathrm{s}$ (Fig. 7). A dissipation function is used to determine thermal capacity of the working chamber in a disk pulse device.

The base for the structural execution of the disk pulse device's working chamber is the following values: $r=0.17 \mathrm{~m}$ and $h=12 \cdot 10^{-3} \mathrm{~m}$.

2. The optimization of parameters for the process of hydrodynamics (velocity and its components) inside the heat generator's working chamber.

The maximum value for the tangential velocity component at the specified geometrical parameters for a working chamber is $V_{\varphi}=1.2 \mathrm{~m} / \mathrm{s}$ (Fig. 6). At the same parameters, a value for the radial velocity component $V_{r}$ is minimum (Fig. 5). The maximum value of $V_{r}=0.5 \mathrm{~m} / \mathrm{s}$ can be achieved at $r=0,17 \mathrm{~m}$ and $h=8 \cdot 10^{-3} \mathrm{~m}$ (Fig. 6). Such a disparity between the components of the specified velocities is associated with the distribution of pressure inside the working chamber of a disk pulse device.

3. The application of a multi-stage system of pulse influence on a heat carrier.

Improving the energy efficiency of blade disk devices (pumps, turbines, compressors) under a multistage (2-fold, 3 -fold and larger) influence on a working medium is explained by the jet theory of fluid flow by Euler, which underlies their operation.

The body of rotating disks hosts the ladle-shaped recesses in the form of turbine blades. Such a profile of disks was selected based on the conducted research, in comparison with other designs of disks [3]. The passage of a working medium through the device's steps increases its pressure that promotes the collapse of cavitation bubbles, the release of additional energy, a decrease in heating time, greater efficiency (Fig. 11).

4. The application of means of automated control over a heating system with the integrated heat generator under the pulse influence on a heat-carrier, which make it possible to rapidly manage the overall process [3].

The obtained experimental indicators for energy efficiency of thermal work of the disk pulse heat generator were compared to the results of thermal work of pulse heat generators from the scientific literature $[3,26]$.

Efficiency of the designed disk pulse heat generator is $16 \%$ larger ( 0.84 versus 0.706 ) than that for the designs of pulse heat generators with a multistage impulse influence reported in the literature [26].

When applying a night tariff, efficiency of the designed heat generator reaches values of $95-98 \%$, due to a decrease in the cost of electricity at night by $50 \%$.

Based on the performed analytical and experimental studies, we have proven the possibility of using the designed disk pulse heat generator for the systems of decentralized heating.

\section{Conclusions}

1. Our analysis of the temperature field distribution in a disk gap of the heat generator has revealed that the maximum temperature of $330 \mathrm{~K}$ corresponds to the impeller's radius $r=0.16-0.17 \mathrm{~m}$ and the height of a disk channel (along the $z$ axis) $h=0.012 \mathrm{~m}$.

2. To determine the optimal geometrical characteristics for a disk pulse heat generator, we varied the impeller's radius $r=0.11 \div 0.17 \mathrm{~m}$; the size of a disk channel $h=(5 \div 12) \cdot 10^{-3} \mathrm{~m}$; 
the flow rate of the heated heat-carrier, $G=0.27 \div 1.1 \mathrm{~kg} / \mathrm{s}$; the installation power $N=7.5 \div 15 \mathrm{kWh}$; the number of rotor's revolutions $n=2,500-3,000 \mathrm{rpm}$. It has been established that the maximum value of efficiency for the single-step disk heat generator does not exceed $\eta=0.76$.
3. Application of the multi-stage pulse influence on a heat-carrier by mounting the second disk on the rotor predetermined an increase in efficiency up to $\eta=0.84$. In this case, the magnitude of gap $c$ between the disks was varied within the range of $(5 \div 15) \cdot 10^{-3} \mathrm{~m}$.

\section{References}

1. Promtov, M. A. (2001). Pul'satsionnye apparaty rotornogo tipa: teoriya i praktika. Moscow, 260.

2. Ved, V., Nikolsky, V., Oliynyk, O., Lipeev, A. (2017). Examining a cavitation heat generator and the control method over the efficiency of its operation. Eastern-European Journal of Enterprise Technologies, 4 (8 (88)), 22-28. doi: https://doi.org/10.15587/ 1729-4061.2017.108580

3. Nikolsky, V., Oliynyk, O., Ved, V., Svietkina, O., Pugach, A., Shvachka, A. (2018). Design and study of the energyefficient unified apparatuses for energytechnological manufacturing. Eastern-European Journal of Enterprise Technologies, 3 (8 (93)), 59-65. doi: https://doi.org/10.15587/1729-4061.2018.132572

4. Pivnyak, G., Dychkovskyi, R., Bobyliov, O., Cabana, E. C., Smoliński, A. (2018). Mathematical and Geomechanical Model in Physical and Chemical Processes of Underground Coal Gasification. Solid State Phenomena, 277, 1-16. doi: https://doi.org/ 10.4028/www.scientific.net/ssp.277.1

5. Nikolsky, V., Yariz, V., Reshetnyak, I. (2017). Improvement of energy efficiency in the operation of a thermal reactor with submerged combustion apparatus through the cyclic input of energy. Eastern-European Journal of Enterprise Technologies, 2 (8 (86)), 39-44. doi: https://doi.org/10.15587/1729-4061.2017.97914

6. Park, J. T., Cutbirth, J. M., Brewer, W. H. (2003). Hydrodynamic Performance of the Large Cavitation Channel (LCC). Volume 2 : Symposia, Parts A, B, and C. doi: https://doi.org/10.1115/fedsm2003-45599

7. Lin, J., Lin, B.-L., Sun, J., Chen, Y.-L. (2016). Modelling hydrodynamic processes in tidal stream energy extraction. Journal of Hydrodynamics, 28 (6), 1058-1064. doi: https://doi.org/10.1016/s1001-6058(16)60711-4

8. Dealy, J. M., Wissbrun, K. F. (2012). Melt rheology and its role in plastics processing: theory and applications. Springer Science \& Business Media, 680

9. Tadmor, Z., Gogos, C. G. (2013). Principles of polymer processing. John Wiley \& Sons, 962.

10. Kuziaiev, I. M. (2008). Modeliuvannia roboty ta proektuvannia ekstruziynykh ahrehativ z rozrobkoiu elementiv SAPR. Dnipropetrovsk: DVNZ UDKhTU, 474.

11. Kuzyaev, I. M., Sviderskiy, V. A., Petuhov, A. D. (2016). Modelirovanie ekstruzii i ekstruderov pri pererabotke polimerov. Ch. 1. Kyiv: NTUU «KPI» Izd-vo «Politekhnika», 412.

12. Momani, S., Odibat, Z. (2006). Analytical solution of a time-fractional Navier-Stokes equation by Adomian decomposition method. Applied Mathematics and Computation, 177 (2), 488-494. doi: https://doi.org/10.1016/j.amc.2005.11.025

13. Girimaji, S. S. (2006). Partially-Averaged Navier-Stokes Model for Turbulence: A Reynolds-Averaged Navier-Stokes to Direct Numerical Simulation Bridging Method. Journal of Applied Mechanics, 73 (3), 413-421. doi: https://doi.org/10.1115/1.2151207

14. Hirsch, C. (2007). Numerical computation of internal and external flows: The fundamentals of computational fluid dynamics. Elsevier, 680. doi: https://doi.org/10.1016/b978-0-7506-6594-0.x5037-1

15. Vetchanin, E. V., Kilin, A. A. (2016). Controlled motion of a rigid body with internal mechanisms in an ideal incompressible fluid. Proceedings of the Steklov Institute of Mathematics, 295 (1), 302-332. doi: https://doi.org/10.1134/s0081543816080186

16. Ma, Y., Liu, H., Zhu, Y., Wang, F., Luo, Z. (2017). The NARX Model-Based System Identification on Nonlinear, Rotor-Bearing Systems. Applied Sciences, 7 (9), 911. doi: https://doi.org/10.3390/app7090911

17. Shevchuk, I. V. (2009). Convective heat and mass transfer in rotating disk systems. Springer, 236. doi: https://doi.org/10.1007/ 978-3-642-00718-7

18. Sheikholeslami, M., Ganji, D. D. (2013). Heat transfer of Cu-water nanofluid flow between parallel plates. Powder Technology, 235, 873-879. doi: https://doi.org/10.1016/j.powtec.2012.11.030

19. Liu, C., Walkington, N. J. (2007). Convergence of Numerical Approximations of the Incompressible Navier-Stokes Equations with Variable Density and Viscosity. SIAM Journal on Numerical Analysis, 45 (3), 1287-1304. doi: https://doi.org/10.1137/050629008

20. Escudier, M. P., Oliveira, P. J., Pinho, F. T. (2002). Fully developed laminar flow of purely viscous non-Newtonian liquids through annuli, including the effects of eccentricity and inner-cylinder rotation. International Journal of Heat and Fluid Flow, 23 (1), 52-73. doi: https://doi.org/10.1016/s0142-727x(01)00135-7

21. Rayher, Yu. L., Rusakov, V. V. (2008). Vraschatel'naya vyazkost' vyazkouprugoy magnitnoy zhidkosti. Kolloidnyy zhurnal, 70 (1), 85-92.

22. Lavrent'ev, M. A., Shabat, B. V. (1973). Metody teorii funktsiy kompleksnogo peremennogo. Moscow: Nauka, 736.

23. Kuziaiev, I. M. (2002). Modeliuvannia neizotermichnykh protsesiv v robochomu obiemi cherviachnykh nasosiv dlia anomalno viazkykh ridyn. Voprosy himii i himicheskoy tekhnologii, 2, 107-112.

24. Kamke, E. (1976). Spravochnik po obyknovennym differentsial'nym uravneniyam. Moscow: Nauka, 576.

25. Antsiferov, S. S., Rusanov, K. E., Afanas'ev, M. S. (2014). Obrabotka rezul'tatov izmereniy. Moscow, 228.

26. Promtov, M. A., Akulin, V. V. (2006). Mechanisms of Heat Generating in the Rotor Impulse Apparatus. Vestnik Tambovskogo gosudarstvennogo tekhnicheskogo universiteta, 12 (2A), 364-369. 